Relations industrielles

Industrial Relations

\title{
Mongrand, Jean-Pierre, Réduction du temps de travail: mode d'emploi
}

\section{Jean Charest}

Volume 54, numéro 2, 1999

URI : https://id.erudit.org/iderudit/051245ar

DOI : https://doi.org/10.7202/051245ar

Aller au sommaire du numéro

Éditeur(s)

Département des relations industrielles de l'Université Laval

ISSN

0034-379X (imprimé)

1703-8138 (numérique)

Découvrir la revue

Citer ce compte rendu

Charest, J. (1999). Compte rendu de [Mongrand, Jean-Pierre, Réduction du temps de travail: mode d'emploi]. Relations industrielles / Industrial Relations, 54(2), 421-423. https://doi.org/10.7202/051245ar

Tous droits réservés @ C Département des relations industrielles de l'Universite Laval, 1999
Ce document est protégé par la loi sur le droit d'auteur. L’utilisation des services d'Érudit (y compris la reproduction) est assujettie à sa politique d'utilisation que vous pouvez consulter en ligne.

https://apropos.erudit.org/fr/usagers/politique-dutilisation/ 
dans leur rapport au travail, qu'elle compare avec celui de mères travailleuses en couple : le défi de la gestion du temps est là aussi crucial. Angelo Soares expose la façon dont les caissières des supermarchés brésiliens et québécois font face à une organisation du travail "sans âme ", la solidarité qui existe entre elles et leurs différentes stratégies pour faire face à des clients violents, au harcèlement sexuel et leur lutte pour conserver le peu de contrôle qu'elles ont sur leur travail. Ana Maria Seifert et coll., recourant à une approche ergonomique pour analyser le travail des caissières de banque à Montréal, font ressortir les exigences au plan physique, mental et émotif de ce travail. Elles s'attachent également aux conséquences des vols de banque en termes de stratégies de support social développées entre les travailleuses suite à un vol ou une agression, et dans la construction du savoir nécessaire à l'accomplissement du travail. Johanne Prévost et Karen Messing, utilisant elles aussi une approche ergonomique, analysent le service 24 heures d'assistance téléphonique à la clientèle d'une entreprise canadienne des communications. Ce travail comporte des caractéristiques d'irrégularité et d'étalement qui représente un grand défi à la programmation d'activités régulières horstravail. Les résultats révèlent un ensemble de stratégies hors-travail dont l'enjeu est la possibilité de rester au travail. Et pour clôturer, Maria de Koninck propose sa lecture personnelle des textes et nous amène à nous questionner sur le sens du travail des femmes, pour les femmes.

L'ensemble de ces textes nous fournit ainsi un panorama très diversifié de ce qu'on regroupe sous un même vocable: stratégies de résistance. Il ressort de ces analyses, à mon sens, une sorte de continuum où la notion de résistance s'apparente d'un côté à la simple stratégie de survie et de l'autre, à une véritable "lutte silencieuse " contre les pressions des rouages de l'organisation. Vers le centre, on aurait les stratégies que l'on pourrait qualifier " d'aménagement " (les stratégies de conciliation famille-travail se trouveraient placées à cet endroit). Autant de formes de "résistance " vécues successivement ou simultanément par les travailleuses. Cet aspect polysémique de la notion de "stratégie de résistance" dans le contexte du travail des femmes m'apparait l'apport le plus substantiel de ce recueil bien construit et riche en données originales.

MARIANNE KEMPENEERS

Département de sociologie Université de Montréal

\section{Réduction du temps de travail : mode d'emploi}

par Jean-Pierre MONGRAND, Paris : Éditions d'Organisation, 1998, 342 p., ISBN

2-7081-2192-8.

S'il est un sujet qui a suscité autant le débat public que les publications en France, au cours des années 90, c'est bien la réduction du temps de travail. Le contexte difficile du marché du travail et l'opposition manifestée par plusieurs acteurs sociaux français à une stratégie de "flexibilisation" tous azimuts des règles de ce marché ont constitué le ferment de la recherche de stratégies autres pour contribuer à la réduction du chômage. C'est dans ce contexte qu'a ré-émergé le sujet de la réduction du temps de travail, considérée comme un mode particulier de régulation du marché du travail et posée comme une des formes de répartition des richesses. La première période de ce débat social, si l'on peut procéder à un tel découpage temporel, a donné lieu à de nombreuses publications à caractère tantôt plus théorique et tantôt plus idéologique. Dans la foulée des mesures législatives des dernières années, dont la loi incitative de juin 1996 (dite "Loi Robien"), puis celle plus directive du 13 juin 1998 (dite "Loi Aubry ") 
qui fixe l'objectif de la durée de la semaine de travail à 35 heures sur l'horizon 2000-2002, laissant aux parties négociantes le choix des modalités concrètes, on a assisté à des publications davantage pragmatiques prenant l'allure du "guide pour réussir la réduction du temps de travail ». Les deux ouvrages ici recensés s'inscrivent précisément dans cette tendance.

L'ouvrage de Jean-Pierre Mongrand est essentiellement un document d'appui à la gestion de l'implantation de la semaine de 35 heures dans les entreprises. C'est un travail bien fait sur le plan pédagogique, qui relève les moments forts de l'histoire française et européenne quant à l'évolution de la durée du travail, qui circonscrit les aspects économiques, légaux et sociaux de la question et qui se concentre surtout sur les facteurs déterminants d'une négociation réussie de ce que l'auteur appelle l'ARTT (aménagement/réduction du temps de travail). D'emblée, il place cet enjeu comme un défi organisationnel qui demande la participation et l'engagement de tous les acteurs de l'entreprise. Ainsi, l'auteur s'adresse-t-il aux cadres qui ont la responsabilité de mener à terme le passage aux 35 heures, mais également aux représentants de la main-d'œuvre (syndicats ou membres des comités d'entreprise) et aux salariés eux-mêmes.

La structure de l'ouvrage en quatorze chapitres repose sur trois thèmes principaux : l'identification de points de repère généraux sur le sujet de l'ARTT (les définitions, les lois, la situation des pays voisins, l'intérêt pour l'entreprise et les résistances au changement); la préparation d'une nouvelle organisation du travail, où l'on décrit les conditions de réussite essentielles au passage aux 35 heures (engagement, mobilisation et attentes dans l'entreprise, la conclusion d'un accord et le cas particulier d'un plan social) ; enfin, la mise en opération d'une nouvelle organisation du travail découlant de l'entente d'ARTT (la préparation du terrain dans l'entreprise et la création d'une nouvelle dynamique sociale interne). Pratiquement tous les chapitres s'accompagnent d'au moins une "fiche outil " présentée en annexe qui a pour fonction de circonscrire les principaux points d'une étape particulière d'ARTT et d'assister les décideurs de l'entreprise. On y retrouve aussi des exemples sur le plan méthodologique permettant de répondre à certaines de ces étapes (grilles d'analyse, construction d'un questionnaire, choix d'indicateurs, etc.).

Pour les aspects opérationnels de l'ARTT, l'ouvrage est donc bien présenté. Il nous semble même que malgré la référence spécifique au contexte français, plusieurs étapes du cheminement proposé pour l'ARTT sont présentées d'une façon suffisamment générale pour pouvoir s'appliquer à d'autres contextes nationaux. L'insistance de l'auteur sur les aspects opérationnels se traduit cependant par une tendance à reprendre la même démarche analytique dans plusieurs chapitres successifs (en fait, les dix derniers chapitres sont articulés autour des mêmes questions posées par l'auteur : "Quels objectifs? Quelle démarche? Quels résultats? Quelles difficultés et comment y faire face? "), ce qui finit par rendre la lecture ennuyeuse, donnant l'impression du déjà vu.

Nous formulons par ailleurs deux critiques plus importantes à l'égard de cet ouvrage. La première est qu'il manque certainement d'appui empirique à plusieurs des énoncés spécifiques qui sont formulés tout au long des chapitres, énoncés qui se présentent comme des formules-conseils en matière d'ARTT. Les expériences concrètes déjà analysées ailleurs et la littérature qui en fait état sont rarement mentionnées par l'auteur. Dès lors, il est légitime de poser la question: d'où viennent les nombreux conseils proposés par l'auteur, que l'on présente comme un consultant expérimenté ? Y a-t-il eu analyse quelconque des cas réussis d'ARTT ? Les déterminants de ces réussites, que semble reprendre l'auteur, répondent-ils à une telle 
analyse ? L'absence de références devient ainsi une faiblesse importante de cet ouvrage dont on se demande s'il n'est pas essentiellement un assemblage personnel de données éparses et d'histoires, au demeurant, inconnues. L'autre critique que nous formulons est que l'auteur est en proie à des contradictions importantes quant à la démarche plutôt consensuelle qu'il propose pour réussir le passage aux 35 heures. Après avoir maintes fois souligné qu'il s'agit d'un projet collectif, d'entreprise, qui nécessite la mobilisation et l'engagement de tous, l'auteur en vient par ailleurs à formuler aux gestionnaires des suggestions du type : comment contourner les résistances syndicales face à l'ARTT ? De tels propos sont pour le moins étranges eu égard à l'approche de fond suggérée auparavant. Notamment, ces propos ne sont pas tout à fait de nature à faire adhérer l'acteur syndical à ce que l'auteur propose comme un "mode d'emploi" pour réussir le passage aux 35 heures!

\section{JEAN CHAREST \\ École de relations industrielles Université de Montréal}

\section{Pensions and Productivity}

par Stuart DORSEY, Christopher CORNWELl et David MACPHERSON, Kalamazoo, Michigan : W.E. Upjohn Institute for Employment Research, 1998, 139 p., ISBN 0-88099-186-0 (alk. paper) et ISBN 0-88099-185-2 (pbk. : alk. paper).

Les régimes privés de retraite jouent un rôle essentiel pour le remplacement des revenus de travail à la retraite. Généralement, ils sont considérés du point de vue de la demande de régimes de retraite. Les travailleurs demandent des régimes de retraite comme source de revenu à la retraite et les employeurs sont indifférents entre payer un salaire et contribuer à un régime de retraite.

Pour les économistes, les régimes de retraite jouent un rôle plus important dans les lieux de travail. En plus d'être une source de revenu pour la retraite, ils peuvent aussi augmenter la productivité au travail et réduire les coûts de maind'œurve. C'est cette perspective du point de l'offre qui est explorée dans le livre Pension and Productivity de Dorsey, Cornwell et Macpherson. Cette publication couvre des aspects théoriques, institutionnels et empiriques et elle introduit de nouveaux résultats suggérant un lien entre les régimes de retraite et la formation offerte aux employés par l'entreprise.

Ce livre se divise en huit chapitres. Dans l'introduction (chapitre 1), les auteurs cherchent à expliquer les pratiques suivies par les employeurs et les employés quand une relation d'emploi est productive et durable, en adressant des questions comme: Quelle est la base économique sous-jacente aux relations d'emploi durables? Comment les pratiques de rémunération et de promotion contribuent-elles à attirer et à motiver les bons employés ? Quand la relation d'emploi est productive, comment les salaires et les avantages sociaux peuvent-ils simultanément permettre des gains de productivité et décourager les mises à pied et les départs?

Le chapitre 2 constitue un survol de la littérature historique et théorique sur les régimes de retraite. Les auteurs font mention des écrits se rapportant à l'apparition des régimes privés de retraite aux États-Unis et, plus particulièrement, des raisons qui ont amené les employeurs à en offrir (réduction du roulement de la main-d'œuvre, attraction des jeunes travailleurs, imposition d'un âge de retraite obligatoire et, plus récemment, report de l'imposition d'une partie de la rémunération). Ils font état de l'évolution des conditions d'acquisition du droit à une rente en cas de cessation de participation. Ils expliquent que les régimes de retraite, 